\title{
LA UTILIZACIÓN DE HERRAMIENTAS DE INVESTIGACIÓN CUALITATIVA EN LA CONSTRUCCIÓN Y DISEÑO DE INDICADORES DE LA PRÁCTICA DE ENFERMERÍA ${ }^{1}$
}

\section{THE USE OF QUALITATIVE RESEARCH TOOLS IN BUILDING AND DESIGNING INDICATORS OF NURSING PRACTICE}

\author{
Patricia Jara C. \\ SANDRA VALENZUELA S.*
}

\begin{abstract}
RESUMEN
La necesidad de diseñar indicadores de la Práctica de Enfermería que permitan evidenciar el valor agregado de la participación de la enfermera/o y que puedan relacionarse con gestión y calidad asistencial en la prestación de servicios de salud, dio origen a una propuesta de investigación que permitió aproximarse a la descripción de la Práctica de Enfermería para luego ir delineando indicadores específicos de ésta. Este artículo tiene por objetivo describir el proceso de delineamiento de indicadores de la Práctica de Enfermería en Unidades de Cuidado Intensivo, utilizando abordajes cualitativos.
\end{abstract}

Palabras clave: Indicadores, Práctica de Enfermería, Investigación Cualitativa.

\begin{abstract}
The need to develop Nursing Practice Indicators to asses the added value of the nurses participation who can relate to management and quality of care in providing health services, led to a research proposal which allowed a closer description of nursing practice and then a specific indicators outlining of this. This article aims to describe the Nursing Practice indicators'delineation process in Intensive Care Units, using qualitative approaches.
\end{abstract}

Key words: Indicators, Nursing Practice, Qualitative Research.

Fecha recepción: 11/03/09 Fecha aceptación: 16/11/09

\section{INTRODUCCIÓN}

Con el fin de establecer indicadores de la Práctica de Enfermería se trabajó en una propuesta que permitiera construir una comprensión desde las bases mismas de la práctica conocida por las enfermeras y enfermeros que trabajan en unidades de cuidado intensivo (UCI), es decir, explorando el ámbito del trabajo de las enfermeras y describiendo en profundidad su práctica. La necesidad de inmersión en la práctica propia de las enfermeras/os requirió la aplicación de un enfoque cualitativo abierto utilizando algunas herramientas como la observación participante, notas de campo, la entrevista en profundidad inicial y de seguimiento y el desarrollo de sesiones con grupos focales de enfermeras de UCI, que más tarde permitiera el análisis y la interpretación de esa información, y la síntesis de una serie de características de la Práctica de Enfermería

\footnotetext{
${ }^{1}$ Proyecto desarrollado y financiado con el apoyo de: Dirección de Investigación de la Universidad de Concepción, Chile DIUC 207.082.038-1.0 y Comisión Nacional de Ciencia y Tecnología FONIS SA07120064

* Candidata Doctoral en Enfermería. Profesora Asociada Universidad de Concepción, Chile. Email:pjara@udec.cl

${ }^{* *}$ Doctora en Enfermería. Profesora Asociada. Universidad de Concepción, Chile. Email: svalenzu@udec.cl
} 
en UCI que más adelante se convertirán en el fundamento para la construcción de los indicadores.

El objetivo de este artículo es describir el proceso de delineamiento de indicadores de la Práctica de Enfermería en Unidades de Cuidado Intensivo, utilizando abordajes cualitativos.

Teniendo presente que se busca que el conocimiento generado permita explicar y comprender los aspectos específicos de la práctica cotidiana de enfermería en UCI, desde el propio referente de las enfermeras y generar desde ese conocimiento una estructura conceptual y originar descripciones de la práctica o praxis misma en el área del cuidado crítico, se está frente a un marco cualitativo inductivo $(1,2)$.

El trabajo de investigación en su primera parte siguió los lineamientos de la investigación cualitativa, específicamente investigación etnográfica, según lo planteado por Michael Agar (3). La etnografía es un método de investigación originalmente desarrollado por antropólogos para estudiar las culturas de determinados pueblos. En el campo de la antropología, Agar plantea que uno de los objetivos de la etnografía es generar relatos que "los miembros de un grupo dicen son posibles interpretaciones de lo que está ocurriendo". El método etnográfico ha sido ampliamente utilizado en el campo de la salud con el fin de estudiar determinadas "culturas" asociadas a ciertas condiciones de salud $(4,5,6)$. En este estudio, el método etnográfico permitió generar relatos de la práctica de enfermería a partir de entrevistas en profundidad y de seguimiento a enfermeras/os de unidades de cuidados intensivos.

Participaron del estudio enfermeras que se desempeñan en dos Unidades de Cuidado Intensivo de la Región del Bío Bío Chile, que consintieron en participar y que respondieron la pregunta: ¿Cómo es la Práctica de Enfermería en UCI?, o simplemente ¿Puedes describir cómo fue tu turno?

Para la recolección de información, ini- cialmente se fijaron reuniones con las enfermeras de estas unidades que coincidió con las reuniones mensuales, donde se presentó el proyecto y se mostró la metodología de trabajo, se planteó la necesidad de realizar las observaciones participantes a las enfermeras(os) en sus turnos sin concertarlas para permitir el azar y la libertad en participar o manifestar abiertamente el deseo de participar durante estas observaciones. Durante tres semanas se realizaron Observaciones Participantes (OP) que consistieron en Visitas de la investigadora a las UCI que duraron entre 4 y $5 \mathrm{~h}$ cada una, con el fin de describir el ambiente de trabajo de las UCI y la dinámica laboral de la unidad; participar con la enfermera(o) del cuidado, si el enfermo consintiera, escuchar las entregas y recepciones de turnos, acompañar a las enfermeras/os durante algunos procedimientos, entre otras. Se solicitó a las enfermeras supervisoras de cada UCI no participar en la convocatoria de enfermeras/os ni en la motivación a participar, para no ejercer algún tipo de coerción sobre las mismas. Durante las OP se contó con una libreta de notas, donde se registraron algunos hechos. Algunas notas son más descriptivas y otras como puntos a recordar; y más tarde se relataba por escrito y guardaba en archivo Word cada una de las anotaciones registradas de estas observaciones. Estas OP permitieron también que las enfermeras manifestaran su consentimiento en participar de las entrevistas y de los grupos focales a desarrollar más tarde. El número de enfermeras/os observadas durante este primer período fue de alrededor de 12 en una unidad y de 5 enfermeras/os en la otra unidad.

Las notas de campo originadas a partir de estas observaciones participantes se hicieron en una libreta de bolsillo por la investigadora como apuntes de hechos que llamaban su atención y posterior a la observación, se escribían detalladamente las situaciones, los hechos, las intervenciones en que participaban las enfermeras que eran observadas. Tanto las notas de campo como las descripciones 
detalladas de las observaciones se mantienen como registro de datos de la investigación. Las notas permitieron a la investigadora destacar aspectos observados que se utilizarán más tarde en las entrevistas en profundidad.

Durante las observaciones, al término o durante el turno, las enfermeras/os manifestaban su consentimiento a ser entrevistadas y se fijaba día, hora y lugar para la entrevista en profundidad, que en la mayoría de los casos correspondió a una visita en su misma unidad en el turno saliente de noche. En este aspecto, hubo mucho interés de participar de las enfermeras tanto en las entrevistas como más tarde en los grupos focales. Cada entrevista en profundidad tuvo una duración de 45 minutos a una hora y fue grabada en condiciones de privacidad, con el consentimiento de la enfermera/o, y su término fue espontáneo, cuando manifestaron no tener nada más que decir. Las grabaciones fueron transcritas verbatim (fielmente) (7) en Word por la investigadora para el análisis posterior. Para las entrevistas en profundidad de seguimiento se escogió aquellas entrevistas que aportaron más elementos de información sobre la práctica de enfermería a juicio de la investigadora. El procedimiento que se siguió fue obtener una impresión de la entrevista y dar una copia a la enfermera entrevistada, esta copia debía ser leída por la enfermera/o dando un tiempo suficiente, que en algunos casos fue una semana y en otros hasta un mes, para luego fijar una nueva sesión de entrevista.

El análisis de la información utilizando las herramientas cualitativas antes descritas se inició tras un corto período luego del comienzo de la recolección de los datos y se hizo siguiendo el análisis temático de Van Manen (8). Primero se analizan las transcripciones y notas de campo como un todo obteniendo una idea general del texto. Para las entrevistas transcritas se realiza un análisis línea por línea y luego frase por frase, buscando elementos descriptores de la práctica de enfermería. En esta fase se utilizó un Diccionario Etimológico (9) que ha sido de mucha utilidad durante el análisis. Finalmente, se estudian los textos en busca de temas emergentes siguiendo el análisis de las etapas previas y se profundiza en los temas emergentes. Se realizó un nuevo análisis de las transcripciones de las entrevistas de seguimiento despúes de mostrar los relatos grabados y luego transcritos verbatim, a las mismas enfermeras.

El análisis de las entrevistas se lleva a cabo por la investigadora y dos enfermeras expertas $^{2}$ ex académicas, estudiosas de la disciplina y la profesión en Enfermería en Chile, y se construye un primer listado de características que corresponde a los temas significativos que describieron las enfermeras/os en las entrevistas. Estos temas significativos que describen elementos de la práctica de enfermería en UCI son seleccionados para trabajarlos con los grupos focales.

Finalmente, con el fin de profundizar hallazgos preliminares y potenciar un primer consenso en el delineamiento inicial de los indicadores de la práctica, como técnica para la recolección de información, se realizaron los grupos focales. Los grupos focales permiten generar un relato detallado de experiencias u opiniones de los participantes de una manera cómoda y no amenazante para ellos (10). Para los grupos focales se realizó un muestreo por conveniencia seleccionando enfermeras que manifestaron su interés por participar en esta actividad de investigación y colaborar en el desarrollo de los enunciados de indicadores. El tamaño de los grupos focales dependió del número total de enfermeras que consintieron participar el día fijado y que por su sistema de cuarto turno pudieron hacerlo. Se trabajó con 7 grupos focales y en promedio cada grupo focal contó con 8 enfermeras/os lo que permitió la conversación y el análisis conjunto.

\footnotetext{
${ }^{2}$ Srta. María Figueroa F. profesora Emérita Universidad de Concepción y Sra. Olga Polanco A. profesora iniciadora de la Especialidad de Enfermería en UCI, Universidad de Concepción.
} 
Aunque se preparó un diagrama o esquema de presentación para cada sesión, finalmente la forma de iniciar el trabajo con los grupos focales, cuyo tema era la Práctica de Enfermería en UCI, varió entre: presentar un relato escrito de una de las entrevistas que se distribuyó entre los asistentes, en que el tema era más significativo y descriptivo; la presentación oral de la temática y finalmente, la escogida por la investigadora, que fue la presentación del subtema o característica con partes de las entrevistas que daban cuenta de ese subtema. Cada sesión de grupo focal fue grabada y cada profesora experta que participó del grupo focal tomó notas escritas; tanto las transcripciones como las notas forman parte de los datos de la investigación.

En las sesiones posteriores se analizó cada característica (alrededor de 30), se discutió sobre el uso de los términos a utilizar para denominar a los enunciados de las dimensiones y las subdimensiones a incluir en cada una. Se consensuó la redacción final y el orden aleatorio para su presentación al conjunto de enfermeras de ambas UCI por separado, quienes hicieron sus comentarios y enriquecieron el análisis, para así poder continuar con la siguiente etapa del estudio.

Para mantener el rigor metodológico en este estudio se han considerado los criterios de credibilidad, aplicabilidad y auditabilidad, que debe cumplir una investigación que utiliza abordajes cualitativos $(11,12,13)$. La recolección y la transcripción fiel de la información fueron realizadas por la investigadora; las entrevistas y el análisis se hicieron manteniendo la expresión de términos utilizados por las enfermeras(os) (14). Considerando los aspectos éticos y de confidencialidad, para el análisis se mantuvo el anonimato de las enfermeras/os entrevistados, cambiando el nombre y en los archivos se otorgó un código numérico a cada entrevista, la lista de los códigos y los nombres se ha guardado por separado. Para la realización de los grupos focales se tomaron partes de los relatos manteniendo fielmente los términos utilizados por las enfermeras en las entrevistas y para la interpretación, se ha levantado un archivo que muestra la concordancia de lo expresado por las enfermeras/os y las características y/o dimensiones y subdimensiones de la Práctica de Enfermería. Además, en los grupos focales como en la presentación final, las enfermeras/ os expresaron su conformidad con la interpretación realizada. Es así como en el desarrollo de uno de los grupos focales, una enfermera planteaba que el relato que acababan de leer era como "una radiografía" de lo que sucede, de cómo es la Práctica de Enfermería en la UCI. Estos registros de las observaciones, notas de campo escritas, entrevistas transcritas, grabaciones digitales de voz y grabaciones de relatos obtenidos en grupos focales, se mantienen resguardados con doble copia por el investigador.

Los resultados de esta primera etapa se complementarán con una segunda etapa que contempla el envío de este listado a enfermeras de diferentes UCI del país para obtener mayor consenso en el delineamiento de los indicadores, para luego en una tercera etapa y final, validar los indicadores que se han construido a partir de las dos etapas anteriores.

\section{CONSIDERACIONES FINALES}

Esta primera fase de abordaje cualitativo abierto, que significó la utilización del enfoque etnográfico y la aplicación de herramientas como: observación participante, notas de campo, entrevistas en profundidad $\mathrm{y}$, posteriormente, el desarrollo de sesiones con grupos focales de enfermeras de UCI, fue un arduo trabajo de acercamiento a estos espacios, el cual no podría haberse realizado sin el importante apoyo de las profesionales del área. Se consideró esencial para explorar el ámbito del trabajo de las enfermeras y ahondar en su práctica. Permitió el delineamiento inicial de indicadores de la práctica 
de enfermería que muestran el valor adicional de la participación de este profesional en la gestión y calidad asistencial de los servicios de salud.

\section{REFERENCIAS}

1. Polit DF, Hungler BP. Investigación Científica en Ciencias de la Salud: principios y métodos. 6 a ed. México D.F.: McGraw-Hill Interamericana; 2000. Pp. 715.

2. Barriga O, Henríquez G. Repensando el conocimiento y la ciencia para la investigación social del siglo XXI: Algunas reflexiones preliminares. Cuadernos de Servicio Social No 1. Universidad San Sebastián. Concepción, Chile. 2004.

3. Agar MH. The professional stranger: an informal introduction to ethnography. San Diego, CA: Academic Press; 1996.

4. Muñoz LA. La enfermedad vino para quedarse. Cienc. enferm., 1995: 1(1), 43-51.

5. Muñoz LA, Jerez V, Calvo MJ. Vivenciando la enfermedad y el cuidado en el ser diabético. Cienc. enferm., 1998: 4(1), 89-98.

6. Alarcón AM, Hermosilla E, Ancavil E, Maniqueo V, Cerda A, Palma E. Una perspectiva cultural de la donación de órganos y tejidos. Cienc. enferm., 2001: 7(2), 67-75.

7. Morse J. Asuntos críticos en los métodos de investigación cualitativos. Medellín, Colombia: Universidad de Antioquia; 2003. Pp. 145.

8. Van Manen M. Researching lived experience. $2^{\text {a }}$ ed. London: The Althouse Press; 1997. Pp. 97-100.

9. Gómez G. Breve Diccionario Etimológico de la Lengua Española. 2a ed. México: Fondo de Cultura Económica; 1998. Pp. 736.

10. Côté-Arsenault D, Morrison-Beedy D. Practical advice for planning and conducting focus groups. Nursing Research, 1999: 48, 280-283.

11. Sandelowski M. The problem of rigor in qualitative research. Advances in Nursing Science 1986: April, 8 (3), 27-37.

12. Sandelowski M. Sample size in qualitative research. Research in Nursing \& Health, 1995: 18, 179-183.

13. Castillo E, Vásquez M. El rigor metodológico en la investigación cualitativa. Colombia Médica 2003: 34(3), 164-167.

14. Gillis A, Jackson W. Research for nurses: methods and interpretation. Philadelphia, PA: F.A. Davis Company; 2002. 